\title{
Conciencia metacognitiva en ingresantes universitarios de ingeniería, arquitectura y ciencias aeronáuticas
}

\section{Metacognitive Awareness in University Graduates in Engineering, Architecture and Aeronautical Sciences}

\author{
Rosario Arias Barahona G. \\ Instituto Peruano de Psicología y Educación, Lima, Perú \\ ORCID: https://orcid.org/0000-0002-9278-5508
}

\section{Ana, S. Aparicio}

Universidad de Sao Paulo, Sao Paulo, Brasil

ORCID: https://orcid.org/0000-0002-2952-2932

*Correspondencia

Email: rosario.arias@psicologiayeducacion.org
Citar como:
Arias Barahona, R., \& Aparicio, A. S. (2020). Conciencia metacognitiva en ingresantes universitarios de ingeniería, arquitectura y ciencias aeronáuticas. Propósitos y Representaciones, 8(1), e272. doi: http://dx.doi.org/10.20511/pyr2020.v8n1.272 


\section{Resumen}

La presente investigación tuvo por objetivo analizar las diferencias en la conciencia metacognitiva y en sus dimensiones al inicio y al final del semestre académico en un grupo de 96 estudiantes de primer ciclo de las carreras de ingeniería, arquitectura y ciencias aeronáuticas de una universidad privada de Lima. También se buscó establecer las diferencias en la conciencia metacognitiva en función del género y la existencia de relación significativa entre la conciencia metacognitiva y sus dimensiones con el rendimiento académico. Esto fue medido a través de las notas promedio finales obtenidas en todas las asignaturas por los estudiantes al acabar el semestre. Se hace uso de un diseño tipo pre test -post test de un solo grupo. El instrumento usado para la evaluación fue el Inventario de Conciencia Metacognitiva de Schraw y Dennison (1994) adaptado en el Perú por Córdova y García (2011). Los resultados arrojan un cambio significativo y favorable en la conciencia metacognitiva y en sus dimensiones de planificación y autorregulación en el post test. No se consiguió establecer relaciones significativas con el género ni con el rendimiento académico.

Palabras Clave: Metacognición; Conciencia metacognitiva; Estudiante universitario; Rendimiento académico.

\section{Summary}

The objective of this research was to analyze the differences in metacognitive awareness and its dimensions at the beginning and end of the academic semester in a group of 96 students in the first cycle of engineering, architecture and aeronautical sciences at a private university in Lima. We also seek to establish differences in metacognitive awareness in terms of gender and the existence of a significant relationship between metacognitive awareness and its dimensions with academic performance. This was measured through the final average grades obtained in all subjects by students at the end of the semester. It makes use of a type of pre-test design subsequent testing of a single group. This was measured through the final average grades obtained in all disciplines by students at the end of the semester. The instrument used for the evaluation was the Metacognitive Consciousness Inventory of Schraw and Dennison (1994) adapted in Peru by Córdova and García (2011).The results found a significant and favorable change in the metacognitive awareness and in its dimensions of planning and self-regulation in the post test; no significant relationships were established with gender or with academic performance.

Keywords: Metacognition; Metacognitive Awareness; University Students; Academic Achievement.

\section{Introducción}

Los avances en psicología cognitiva han cambiado las concepciones del proceso enseñanza aprendizaje, poniendo énfasis en los procesos internos de quien aprende y reconociendo el papel activo que el aprendiz tiene en sus propios procesos de aprendizaje. Hoy se habla de tener estudiantes cada vez más conscientes y responsables de sus capacidades, procesos y resultados de aprendizaje; que adquieren capacidades de aprendizaje que les facilitan adaptarse mejor a las exigencias de los estudios universitarios y las nuevas demandas laborales y tecnológicas. Esto plantea ayudarlos a que logren aprender a aprender de forma autónoma, autorregulada y orientarlos a reflexionar sobre sus propias estrategias de aprendizaje, favoreciendo el aprendizaje significativo, la memorización comprensiva, el juicio crítico en su rol de estudiante y futuro profesional (Naigeboren \& Caram, 2004). 
El proceso educativo centrado en el aprendizaje plantea un modelo educativo que responda a las necesidades del estudiante, un perfil del estudiante que tome en cuenta su desarrollo desde las dimensiones cognitiva, socio afectiva y actitudinal; asimismo un docente en el rol de mediador o facilitador del aprendizaje, un nivel de cambio en los estudiantes con una forma de hacer que involucre estrategias cognitivas y metacognitivas, así como innovaciones en la elaboración de las tecnologías de la información.

De acuerdo a estudios como los de Córdoba y Marroquín (2018), Abdellah (2015), Sonowal y Kalita (2017), Owo y Ikwut (2017) la metacognición juega un papel importante en la educación porque ayuda a los alumnos a ser capaces de desarrollar un plan, monitorear y evaluar su efectividad, eso significa que la metacognición ayuda al alumno a participar más en el proceso de aprendizaje, y de acuerdo con Hernández y Camargo (2017), la instrucción basada en estrategias metacognitivas es un buen predictor de un aprendizaje eficaz.

La Metacognición es un constructo de gran importancia para el aprendizaje, que comprende el conocimiento y regulación de nuestras propias cogniciones, así como de los procesos mentales de percepción, atención, memoria, comprensión, comunicación y los que subyacen a la lectura y escritura, en el sentido de lo qué significan, cómo se realizan, cuándo hay que usar uno u otro y cuáles factores ayudan o interfieren su operatividad. De acuerdo con Cantillo, De la Hoz y Cerhiaro (2014) la actividad metacognitiva se aborda como problema de investigación en el campo de la psicología cognitiva y educativa, a partir del interés por conocer acerca de los procesos cognitivos de orden superior que las personas llevan a cabo cuando aprenden, razonan, resuelven problemas o comprenden un texto.

Hacia la década de los 70, Flavell (1979) dio origen al término metacognición e inició su investigación conceptuándola como el conocimiento que uno tiene acerca de sus propios procesos y productos cognitivos, a la monitorización, la regulación y ordenación de dichos procesos en relación con los objetos cognitivos, datos o información sobre los cuales ellos influyen, normalmente al servicio de un objetivo o meta relativamente concreta.

Pacheco (2012) basándose en Burón (1993) expone que una de las características fundamentales de la metacognición es su carácter motivacional; el estudiante metacognitivo reconoce que aprender requiere de esfuerzo y en tal sentido la motivación es considerada como la que cierra el ciclo completo: el proceso, el control, el juicio y la decisión metacognitiva que provoca la autorregulación. En tal sentido, las actividades metacognitivas suponen mecanismos de autorregulación y de control que le sirven al sujeto cuando se muestra activo en la resolución de problemas.

Meza y Lazarte (2007) definen la metacognición como el grado de conciencia que la persona (el estudiante) aplica deliberadamente sobre sus procesos, contenidos o actividades cognitivos con el objetivo de controlarlos. Distinguen el significado del término conciencia, así como del control. Utilizan dos acepciones de la conciencia, una, el darse cuenta de un proceso, un contenido o una actividad cognitivos y la otra, el darse cuenta del grado de compromiso que se pone en ello y de que se está reflexionando "mirando hacia adentro". Por otro lado, el control significa regular, dirigir, canalizar, cuidar que se tome y retome el camino que ayudará que se logre el objetivo.

Por su parte, Huertas, Vega y Galindo (2014) señalan que la metacognición se refiere a los procesos reflexivos que las personas hacen sobre su propio conocimiento, así como al conocimiento que tienen acerca de la propia actividad cognitiva, éste le permite, cuando realiza alguna tarea, por una parte, monitorear lo que hace, esto es ejercer una supervisión mientras avanza en su actividad y, por otra, dirigir el curso de sus cogniciones, es decir, ejercer control. Asimismo, destacan que se puede identificar en esta definición dos elementos integrantes de la 
metacognición, la conciencia del propio conocimiento y el control o regulación que sobre éstos se ejerce.

La metacognición es una de las manifestaciones más importantes del aprender a aprender, que comprende autodirigir el aprendizaje y transferirlo a otros ámbitos de la vida. Está constituido por tres saberes: (a) Saber los procesos mentales implicados en el aprendizaje, el real conocimiento sobre lo que uno sabe y lo que desconoce y el conocimiento sobre las distintas estrategias posibles para afrontar la tarea, (b) Saber hacer estrategias de planificación para resolución de una tarea, estrategias de supervisión de las acciones que uno está desarrollando, estrategias de evaluación del resultado y del proceso que se ha llevado a cabo y, (c) Saber ser, que implica motivarse para aprender, sentirse protagonista del proceso y del resultado de su aprendizaje, tener la percepción de autoeficacia y confianza en sí mismo.

Según Meza y Lazarte (2007) la metacognición y las estrategias de aprendizaje forman una alianza educativa. Por un lado, la metacognición prepara la puesta en marcha de las estrategias de aprendizaje al dirigir la conciencia a procesos y contenidos que necesitan ser intervenidos y también las focaliza. Por otro lado, la misma metacognición puede convertirse en estrategia, como en el caso de los procedimientos de lectura comprensiva en los que el control consciente antes, durante y después de la lectura son esenciales. Tanto la metacognición como las estrategias cognitivas promueven el autocontrol y éste implica: saber auto-observarse, saber autoevaluarse y saber auto incentivarse.

Las estrategias metacognitivas de aprendizaje son el conjunto de acciones orientadas a conocer las propias operaciones y procesos mentales (qué), saber utilizarlas (cómo) y saber readaptarlas y/o cambiarlas cuando así lo requieran las metas propuestas. Se desenvuelven por medio de los procesos de planificación, autorregulación y evaluación (Osses \& Jaramillo, 2008). Para estos autores, la planificación es el análisis y reflexión de la selección de estrategias adecuadas y la determinación de pasos lógicos, tomando en cuenta factores personales implícitos y los condicionantes del entorno con el fin de poder programar una acción eficaz y, la autorregulación es la revisión de la ejecución de las tareas, detectando dificultades en el curso de acción para su adecuación a la planificación propuesta o a nuevas vías no previstas a fin de optimizar los resultados.

Por otro lado, la evaluación comprende la necesidad de hacer un proceso de valoración general, que incluye todas las actividades físicas y mentales que se han llevado a cabo para concluir el trabajo y entre ellas: evaluar el aprendizaje, los recursos, la funcionalidad, la adecuación, las alternativas, las estrategias, las técnicas empleadas, elaborar conclusiones, ventajas así como inconvenientes (Naigeboren \& Caram, 2004) y se debe enfatizar que la evaluación es para aprender y permitir al estudiante ser autónomo y autorregular su proceso de aprendizaje mientras planifica y distribuye el tiempo para el estudio, da orden y estructura los pasos para completar una actividad evaluativa (Bogantes \& Palma, 2016).

Las estrategias metacognitivas se emplean para planificar, supervisar y evaluar la aplicación de las estrategias cognitivas y se constituyen en su apoyo, por apuntar a aumentar y mejorar los productos de nuestra actividad cognitiva, favoreciendo la codificación y almacenamiento de información, su recuperación posterior y utilización en la solución de problemas. Psicólogos y educadores consideran que el conocer las estrategias metacognitivas es un aspecto decisivo en el desarrollo del pensamiento reflexivo, del aprendizaje autónomo y de la construcción del conocimiento (Jiménez, Puente, Alvarado, Arrebillaga \& Durante, 2009).

De acuerdo con diferentes investigadores como Osses y Jaramillo (2008); Campo, Escorcia, Moreno y Palacio (2016) se hace referencia a la importancia de desarrollar habilidades metacognitivas en los estudiantes porque está relacionada con el logro académico. El aprendizaje 
autorregulado y el desarrollo del pensamiento crítico, el conocimiento metacognitivo que posee un individuo, permite sentirse más motivado y a medida que es más consciente de los mecanismos de autorregulación de los procesos que utiliza para el aprendizaje, aprende mejor y más eficazmente.

Doganay y Demir (2011) encontraron diferencias significativas entre el uso de estrategias metacognitivas en estudiantes de carreras docentes que tenían alto y bajo logro académico; el uso de dichas estrategias estuvo asociado con alto desempeño. Por su parte, Thornberry (2008) investigó la relación entre el uso de estrategias metacognitivas, la motivación académica y el rendimiento académico en 116 estudiantes universitarios ingresantes al primer ciclo de estudios de una universidad privada de Lima. Se elaboró la Escala de Motivación Académica que evalúa las dimensiones motivacionales de autoeficacia académica, atribuciones causales y motivación de logro. Los resultados revelaron que existen correlaciones positivas entre el rendimiento académico y las estrategias metacognitivas, aunque no lograron ser predictivas. En cambio, la motivación académica logra predecir el rendimiento en un $12.6 \%$. Se concluye que la motivación académica sí resulta predictiva del rendimiento académico, mientras las estrategias metacognitivas no.

Arteta y Huaire (2016) tuvieron como propósito establecer la relación que existe entre estrategias metacognitivas y concepciones de aprendizaje o ideas de carácter intuitivo que poseen tanto profesores como estudiantes respecto de los procesos, las condiciones y los resultados involucrados en la enseñanza y el aprendizaje. La investigación adoptó un diseño descriptivo correlacional y se llevó a cabo con 369 estudiantes del curso de Bienestar total (Total Wellness) de una universidad privada de Lima. Los instrumentos utilizados fueron el cuestionario de autoreporte de O'Neil y Abedi (1996) que contiene 20 ítems y el cuestionario de auto-reporte CONAPRE, diseñado y validado por Martínez (2004), que consta de 15 ítems. A partir del análisis de los datos se concluye que, existe una relación estadísticamente significativa entre las estrategias metacognitivas y concepciones de aprendizaje.

Moreano y Roca (2014) realizaron un estudio descriptivo-transversal para identificar cuáles eran las estrategias cognitivas y metacognitivas que se aplicaban en el curso de redacción, enseñado a todos los ingresantes del área de humanidades de una universidad privada de Lima. El análisis de un cuadro que elaboraron detallaba los logros y actividades del curso, dentro del cual se articulaban los logros por unidad y por sesión de clase, les permitió observar que, en la mayor parte de las sesiones, la actividad realizada ayudó a alcanzar el logro predeterminado. El instrumento empleado fue una matriz de estrategias cognitivas y metacognitivas de la composición de textos. Concluyeron que predominaba el uso de las estrategias cognitivas, especialmente las de planificación, para organizar y producir los textos, mientras que la reflexión metacognitiva se hizo de forma oral y de manera intuitiva.

Guerrero (2017) se propuso determinar la relación entre las estrategias metacognitivas de lectura del nivel inferencial con la comprensión de textos argumentativos en 100 estudiantes del primer ciclo que iniciaban la carrera de Administración y Negocios Internacionales, pertenecientes a una Facultad de Ciencias Empresariales de Lima. Los resultados obtenidos determinaron que las estrategias metacognitivas para la comprensión lectora influyen significativamente a nivel inferencial en textos argumentativos.

Cantillo et al. (2014) propusieron estudiar los niveles de actividad metacognitiva en estudiantes universitarios colombianos, estableciendo diferencias en función de variables como la edad y semestre académico. Se utilizó el Cuestionario de Auto reporte de Actividad Metacognitiva. Participaron 95 estudiantes varones y mujeres, que cursaban el primero, quinto y décimo semestre en un programa de psicología de una universidad pública. Los resultados permiten establecer que la actividad metacognitiva, en relación tanto con el semestre como con 
la edad en la muestra estudiada, se ubica en un nivel medio bajo. En ninguno de los semestres, ni en los rangos de edad se registran niveles altos en los componentes de conciencia, control y autopoiesis. No se encontró correlación significativa entre actividad metacognitiva y semestre académico cursado, como tampoco con la edad.

Campo et al. (2016) estudiaron los niveles de metacognición con relación a la producción de trabajos escritos, en 462 estudiantes universitarios de psicología de primer a sexto ciclo, la mitad colombianos y la mitad franceses, con edad promedio de 18.8 y 20.1 años, respectivamente, quienes respondieron un cuestionario de autorreporte sobre metacognición compuesto por dos escalas: conocimientos metacognitivos y estrategias de planeación. Para ello construyeron un cuestionario en base al Inventario de Conciencia Metacognitiva (MAI) de Shcraw y Denninson (1994) para medir la conciencia metacognitiva y al cuestionario de Escorcia y Fenouillet (2011) que mide la metacognición en la escritura. Hallaron una correlación positiva entre el nivel de rendimiento y los conocimientos metacognitivos.

Algunos investigadores sostienen que la metacognición se correlaciona significativamente con el rendimiento académico o los logros de los estudiantes (Campos et al, 2016; Owo \& Ikwut, 2017; Hernández \& Camargo, 2017; Bogdanović et al., 2015). En esa dirección, las estrategias cognitivas van a ayudar a los estudiantes a desarrollar un conjunto de habilidades metacognitivas para autorregular su aprendizaje y retener información más tiempo y un mejor desempeño.

Ramírez, Olarte y García (2013) realizaron un estudio destinado a determinar si las estrategias de aprendizaje autorregulado influyen en el rendimiento académico de estudiantes de ingeniería eléctrica y electrónica de la Universidad Nacional de Colombia. Trabajaron con 396 estudiantes, que fueron encuestados sobre su uso de estrategias de aprendizaje utilizando el Cuestionario de Estrategias de Aprendizaje y Motivación CEAM II; asimismo, se propusieron encontrar si existía relación entre las puntuaciones obtenidas en el cuestionario y las calificaciones obtenidas en dos cursos del plan de estudios de ingeniería. Encontraron que algunas estrategias están relacionadas con el logro académico de ambos cursos, mientras que otras solo influían en uno de ellos.

Escanero, Soria, Escanero y Guerra (2013) analizaron la relación existente entre los estilos de aprendizaje y el conocimiento metacognitivo con el rendimiento académico en 174 estudiantes españoles de fisiología de segundo curso de una facultad de medicina. Aplicaron el Cuestionario Honey-Alonso de Estilos de Aprendizaje (CHAEA) y el Inventario sobre Estrategias Metacognitivas de O'Neil y Abedi (1996). El rendimiento académico se obtuvo a partir de la nota final alcanzada por cada uno de los alumnos. Los resultados muestran que la planificación como proceso de la metacognición y la nota obtenida se correlacionaron significativamente $(\mathrm{p}<.05)$.

Pacheco (2012) estableció la relación entre las estrategias metacognitivas y el rendimiento académico en la asignatura de metodología del aprendizaje e investigación, en 109 estudiantes que cursaban el primer ciclo de ingeniería civil de una universidad nacional de Lima. Utilizó la Encuesta de estrategias metacognitivas de O’Neil y Abedi (1996). Los resultados muestran correlación positiva media entre las variables de estudio. Encontró que existe un predominio metacognitivo al nivel promedio, aunque en el nivel superior destacan los varones más que las mujeres. Al comparar los niveles promedio y superior de las dimensiones de autoconocimiento y autorregulación con los del rendimiento académico, encuentra un empate porcentual en el uso de las estrategias metacognitivas, que aparentemente lleva a plantear que los estudiantes que tienen el mismo nivel de rendimiento académico utilizan en similar nivel las estrategias metacognitivas.

Broc (2012) estudió la influencia relativa que ciertas variables metacognitivas y volitivas ejercen sobre el rendimiento académico en 113 estudiantes españoles de Bachillerato, con edades entre los 16 y 20 años. Utilizó el inventario de estrategias de estudio y aprendizaje (LASSI) de 
Weinstein, Schulte y Palmer (1987), el de Estrategias Volitivas Académicas (IEVA) de Mccann y Turner (2004) y un Cuestionario de Gestión de tiempo. Analizó las diferencias significativas encontradas en el rendimiento académico final en función de la modalidad elegida (Ciencias versus Letras) encontrando resultados a favor de los primeros.

Arias, Zegarra y Velarde (2014), indagaron acerca de la relación de los estilos de aprendizaje según el modelo de Kolb (1979), con la metacognición y sus tres dimensiones de autoconocimiento, autorregulación y evaluación. Los participantes fueron 273 estudiantes de psicología de tres universidades de Arequipa-Perú. Los instrumentos utilizados fueron el Inventario de Estilos de Aprendizaje de Kolb adaptado por Ezcurra (1992) y el Inventario de Estrategias Metacognitivas de O'Neil y Abedi (1996). Los resultados indican que existen altos porcentajes de estudiantes con niveles bajos de metacognición, autoconocimiento, autorregulación y evaluación. Un análisis de varianza señala que los estilos de aprendizaje no tienen efecto sobre la metacognición ni en sus dimensiones.

Ramírez, Olarte y García (2014) investigaron acerca del nivel de utilización de estrategias de aprendizaje de 229 estudiantes de primer semestre de Ingeniería Eléctrica e Ingeniería Electrónica de una universidad colombiana. Los datos sobre la utilización de estrategias de aprendizaje se obtuvieron por medio del cuestionario de estrategias de aprendizaje y motivación (CEAM II) y fueron recolectados con dos grupos similares de estudiantes, en los años 2013 y 2014. Los resultados indican que la estrategia de regulación del esfuerzo es significativamente más usada por los estudiantes participantes; mientras que las estrategias de organización de ideas, el aprendizaje con pares y la administración del tiempo de estudio son escasamente utilizadas.

En el presente estudio, de carácter descriptivo, comparativo y correlacional, nos propusimos analizar en una muestra de estudio, compuesta por un grupo de estudiantes de ingeniería, arquitectura y ciencias aeronáuticas de una universidad privada de Lima, el cambio en la conciencia metacognitiva y sus dimensiones entre el pre test y el post test, en tal sentido, se comparó de manera descriptiva los niveles de la conciencia metacognitiva y sus dimensiones en el pre test y post test, se estableció las diferencias en la conciencia metacognitiva por género y se relacionó la conciencia metacognitiva y sus dimensiones con el rendimiento académico evaluado al final del semestre académico. Adicionalmente se verificó tanto la validez como la confiabilidad del instrumento utilizado para la investigación.

\section{Método}

\section{Participantes}

En el estudio participaron 123 ingresantes universitarios de ingeniería, arquitectura y ciencias aeronáuticas de los cuales se obtuvo una muestra final de 96 participantes, 72 varones (75\%) y 24 mujeres $(25 \%)$. La selección de la muestra fue de tipo intencional obtenida en la Facultad de ingeniería y arquitectura de una universidad privada de Lima, en el marco de un curso introductorio de nivelación, previo al inicio del primer ciclo, semestre 2016- I.

\section{Instrumentos}

\section{Inventario de Conciencia Metacognitiva (ICM).}

El Inventario de conciencia metacognitiva (Metacognitive Awareness Inventory) fue elaborado por Schraw y Dennison (1994), en la Universidad de Nebraska de Lincoln, para identificar habilidades metacognitivas en los sujetos y para mostrar la relación de la metacognición con el logro de aprendizaje. Fue traducido y adaptado para Perú por Fernando Córdova y Augusto García (2011) con estudiantes de una universidad privada de Lima. La adaptación peruana del inventario 
de conciencia metacognitiva tiene un total de 80 ítems, se le agregaron 28 ítems de los 52 que tiene la versión original, distribuidos en las diferentes dimensiones de la prueba.

Las dimensiones del inventario son tres y se refieren a los procesos que integran la conciencia metacognitiva: (a) planificación con 24 ítems, autorregulación con 33 ítems y evaluación con 23 ítems, respectivamente.

La dimensión de planificación responde a la pregunta ¿Qué actividades cognitivas voy a realizar? Evalúa si el examinado lleva a cabo las estrategias de la selección de estrategias adecuadas, la determinación de los pasos lógicos a seguir, asignación de recursos, nivel de conocimientos previos y hacer predicciones antes de comenzar la tarea. Entre las capacidades específicas que generan estrategias de planificación, evalúa si el examinado predice, identifica, selecciona, intuye, anticipa, descubre, representa, describe, organiza, inventa, clasifica, utiliza, crea, formula, propone, proyecta, diseña, plantea, determina, entre otras.

La dimensión de autorregulación trata de responder a la pregunta ¿Cómo lo estoy haciendo? Evalúa si el examinado lleva a cabo las estrategias de revisión de la ejecución de las tareas, detecta las posibles dificultades en el curso de acción y utiliza las estrategias de modo flexible. Entre las capacidades específicas que generan estrategias de autorregulación, evalúa si el examinado controla, discrimina, analiza, interpreta, sintetiza, argumenta, sistematiza, diagrama, integra, prioriza, reflexiona, comprende, discrepa, demuestra, relaciona, categoriza, generaliza, produce, contrasta, extrapola, intuye, reproduce, etc.

La dimensión de evaluación contesta a la pregunta ¿Qué también lo estoy haciendo? Evalúa si el examinado lleva a cabo las estrategias de apreciación de los procesos reguladores, la determinación del valor de los productos logrados, la definición de las metas y objetivos logrados, la evaluación de la eficacia de las estrategias utilizadas, la modificación del plan de acción y la retroalimentación constante. Entre las capacidades específicas que generan estrategias de evaluación, evalúa si el examinado enjuicia, autoevalúa, retroalimenta, juzga, revisa, valora, reconstruye, asume, interioriza, discrepa, propone, resume, propicia, entre otras.

El inventario plantea preguntas al examinado acerca de cómo hace sus tareas académicas, cómo resuelve problemas, cuáles son sus estrategias de estudio, entre otras. Presenta una escala de cinco categorías para responder si cada frase del inventario corresponde realmente a su peculiar forma de actuar, en la que tendrá que elegir una de ellas: A) No; B) A veces; C) Postura intermedia; D) A veces sí; E) Sí. La puntuación va de 1 a 5 de acuerdo con la frecuencia del comportamiento metacognitivo. En la evaluación de la conciencia metacognitiva en base a los puntajes obtenidos, se puede ubicar a los examinados según su desempeño en niveles Muy bajo, Bajo, Promedio y Bajo.

Las propiedades psicométricas del Inventario fueron verificadas por Córdova y García (2011). La validez de criterio la establecieron en base a correlaciones entre el constructo general y las dimensiones, obteniéndose correlaciones significativas al nivel 0.01 y la confiabilidad usando el Alpha de Cronbach, que mostró un coeficiente de 0.95 en la conciencia metacognitiva.

Para el presente estudio replicamos lo hallado por estos autores, en cuanto a la validez de criterio, encontrando también tanto en el pre test como en el post test correlaciones significativas al 0.01 , como es mostrado en la tabla 1 . 
Tabla 1.

Correlaciones entre conciencia metacognitiva y sus dimensiones en el pre test y post test $(n=96)$

\begin{tabular}{lcccccccc}
\hline & \multicolumn{3}{c}{ Pre Test } & \multicolumn{5}{c}{ Post Test } \\
\hline & Metac & Dpla & Dauto & Deva & Metac & Dpla & Dauto & Deva \\
\hline Meta & 1 & $0.92^{* *}$ & $0.96^{* *}$ & $0.95^{* *}$ & 1 & $0.93^{* *}$ & $0.96^{* *}$ & $0.95^{* *}$ \\
Dpla & & 1 & $0.80^{* *}$ & $0.82^{* *}$ & & 1 & $.835^{* *}$ & $0.85^{* *}$ \\
Dauto & & & 1 & $0.88^{* *}$ & & & 1 & $0.86^{* *}$ \\
Deva & & & & 1 & & & & 1 \\
\hline
\end{tabular}

**. Correlación significativa a nível .01

Metac: conciencia metacognitiva; Dpla: Dimensión Planificación; Dauto: Dimensión Autorregulación, Deva: Dimensión Evaluación

De igual forma, la confiabilidad del Inventario en nuestra muestra de estudio fue verificada aplicando el Alfa de Cronbach. Se obtuvo un coeficiente de 0.94 y 0.95 en la conciencia metacognitiva en el pre y post test respectivamente, que mostró una alta consistencia interna. Además, en todas sus dimensiones el coeficiente fue superior a 0.81. En tal sentido, la consistencia interna encontrada es adecuada (ver tabla 2).

Tabla 2.

Valores de confiabilidad Alpha de Cronbach en la conciencia metacognitiva y sus dimensiones en el pre test y post test $(n=96)$

\begin{tabular}{lcc}
\hline & \multicolumn{2}{c}{ Alpha de Cronbach } \\
\cline { 2 - 3 } & Pre Test & Post Test \\
\hline Conciencia Metacognitiva & 0.94 & 0.95 \\
Dimensión Planificación & 0.82 & 0.84 \\
Dimensión Autorregulación & 0.87 & 0.90 \\
Dimensión Evaluación & 0.85 & 0.88 \\
\hline
\end{tabular}

Por los índices de confiabilidad obtenidos en la adaptación peruana del Inventario de Conciencia Metacognitiva y en la muestra de participantes del presente estudio, se comprueba la buena consistencia interna del instrumento utilizado.

\section{Rendimiento Académico.}

En nuestro estudio hemos considerado el rendimiento académico como la medida en que un estudiante ha alcanzado sus logros educativos, interpretado por la nota promedio general obtenida al final del semestre, que resume las calificaciones, en sistema vigesimal, alcanzadas por los estudiantes en las ocho asignaturas que se imparten durante el primer ciclo. En las carreras de ingeniería comprende un $20 \%$ de cursos de matemática y ciencias básicas, $65 \%$ de humanidades y $15 \%$ de pre-especialidad; en ciencias aeronáuticas un $20 \%$ de cursos de matemática y ciencias básicas, $55 \%$ son de humanidades y un $25 \%$ de pre- especialidad; similar distribución se da en arquitectura. En los cursos de matemáticas, ciencias básicas y pre-especialidad incorporan en la evaluación cuatro prácticas calificadas y examen final; con excepción de arquitectura que presenta un curso cuyo avance se evalúa semanal (Taller I). Los cursos de humanidades comprenden trabajos e informes escritos, exposiciones, examen parcial y final. 


\section{Procedimiento}

La recogida de datos del pre test se llevó a cabo en el mes de febrero de 2016 y el pos test en el mes de junio del mismo año, antes del inicio de los exámenes de final de semestre. La administración adoptó los criterios estándares de aplicación de los instrumentos. Tanto en el pre test como en el post test se hizo de forma colectiva; en el pre test, se aplicó en simultáneo en cuatro aulas a cargo de uno de los investigadores y tres profesores entrenados. En el post test, un investigador se responsabilizó de la aplicación. No hubo tiempo límite en la administración del instrumento, insumiendo 35 a 40 minutos aproximadamente. Se informó a los participantes que su aceptación en la aplicación de los cuestionarios indicaba su consentimiento para que los datos fueran utilizados para fines de análisis estadísticos, siendo objeto posterior de presentación académica.

Durante el semestre académico los profesores responsables de las disciplinas impartieron sus clases basándose en la metodología establecida en los sílabos, cuyo peso está dado en razón a la naturaleza de la asignatura. Comprende la exposición del docente y la interacción con el estudiante, trabajos de aplicación individual y de grupo. Método de discusión guiada. Conducción del grupo para abordar situaciones para llegar a conclusiones y recomendaciones. Debate y diálogo en el aula. Método de demostración-ejecución: se utiliza para ejecutar, demostrar, practicar y retroalimentar lo expuesto.

Los análisis del estudio fueron realizados con los estudiantes que habían cursado las ocho (8) asignaturas que comprende el plan de estudios para el primer ciclo y que totalizan 23 créditos. La muestra final de estudio que cumplió con este criterio fue de 96 evaluados.

\section{Resultados}

\section{Análisis de Cambios}

Para analizar el cambio en la conciencia metacognitiva y sus dimensiones, entre el pre test y el post test hemos utilizado una prueba $t$ de Student para muestras apareadas. Los resultados indican que hay un cambio significativo favorable en el post test, a nivel de $\mathrm{p}>0.05$, en la conciencia metacognitiva y las dimensiones de planificación y autorregulación, con excepción de evaluación, en el que la diferencia no es significativa.

Se puede observar en la Tabla 3, que los promedios tanto en la conciencia metacognitiva como en sus dimensiones se elevan en el post test.

Tabla 3.

Cambio en los promedios de la conciencia metacognitiva en el pre test y el post test $(n=96)$

\begin{tabular}{|c|c|c|c|c|c|c|c|}
\hline & \multicolumn{2}{|c|}{ Pretest } & \multicolumn{2}{|l|}{ Postest } & \multirow{2}{*}{$\begin{array}{c}\mathrm{t} \mathrm{de} \\
\text { muestras } \\
\text { apareadas }\end{array}$} & \multirow[b]{2}{*}{$\mathrm{gl}$} & \multirow[b]{2}{*}{ Sig. } \\
\hline & Media & D.E & Media & D.E & & & \\
\hline Conciencia Metacognitiva & 305.69 & 39.64 & 312.55 & 40.66 & 2.00 & 95 & $0.048 *$ \\
\hline Planificación & 90.28 & 12.15 & 92.61 & 11.90 & 2.16 & 95 & $0.033 *$ \\
\hline Autorregulación & 127.96 & 16.38 & 131.11 & 16.99 & 2.02 & 95 & $0.046^{*}$ \\
\hline Evaluación & 87.49 & 13.43 & 88.82 & 13.94 & 1.05 & 95 & 0.297 \\
\hline
\end{tabular}

p>*.05: diferencia significativa 


\section{Comparación descriptiva de frecuencias en los niveles de conciencia metacognitiva}

Una comparación descriptiva de frecuencias y porcentajes en los niveles de conciencia metacognitiva, y sus dimensiones en el pre test y post test, evidencia que en general los participantes están en nivel promedio con muy pequeñas variantes en los niveles anteriores y posteriores.

En cuanto a la conciencia metacognitiva, tanto en el pre test como en el post test un 4.2 presenta un nivel muy bajo, mientras que un $11.5 \%$ tiene un nivel muy alto en el pre test y un $12.5 \%$ en el post test (ver tabla 4 y figura 1 ).

Tabla 4.

Porcentajes según niveles de conciencia metacognitiva Pre y Post test ( $n=96)$

\begin{tabular}{lcccc}
\hline $\begin{array}{l}\text { Nivel Conciencia } \\
\text { Metacognitiva }\end{array}$ & Pre test & \multicolumn{3}{c}{ Post test } \\
\hline & Frecuencia & $\%$ & Frecuencia & $\%$ \\
\hline Muy Bajo & 4 & 4.2 & 4 & 4.2 \\
Bajo & 9 & 9.4 & 11 & 11.5 \\
Promedio & 72 & 75.0 & 69 & 71.9 \\
Muy Alto & 11 & 11.5 & 12 & 12.5 \\
\hline
\end{tabular}

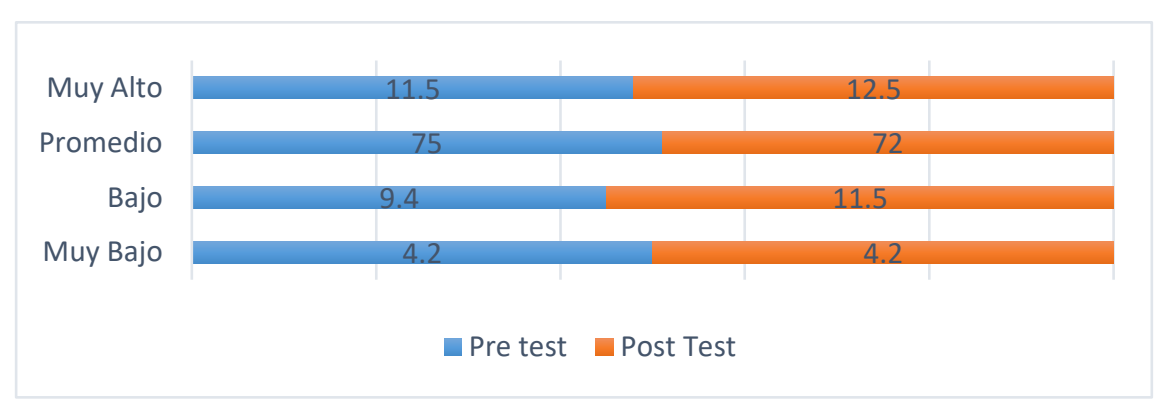

Figura 1. Comparación de frecuencias en los niveles de conciencia metacognitiva Pre- test y Post test

Respecto de las dimensiones de la conciencia metacognitiva, en planificación hay un $5.2 \%$ en el nivel muy bajo en el pre test frente a un $3.1 \%$ en el post test y un $14.6 \%$ en el nivel muy alto en el pre test y un 12.5 en el post test. En la dimensión autorregulación hay un $3.1 \%$ en el nivel muy bajo tanto en el pre test como en el post test y un $8.3 \%$ en el nivel muy alto en el pre test y un $12,5 \%$ en el post test. Por último, la dimensión evaluación hay un $1.0 \%$ en el nivel muy bajo en el pre test frente a un 3.1 en el post test y un $8.3 \%$ en el nivel muy alto en el pre test y un $12.5 \%$ en el post test (ver tabla 5). 
Tabla 5.

Comparación de frecuencias en los niveles de las dimensiones de la conciencia meta-cognitiva Pre y Post test

\begin{tabular}{llcccc}
\hline \multirow{2}{*}{$\begin{array}{l}\text { Dimensiones de la Conciencia } \\
\text { Metacognitiva }\end{array}$} & Niveles & Pre Test & \multicolumn{3}{c}{ Post Test } \\
\cline { 3 - 6 } & & Frecuencia & $\%$ & Frecuencia & $\%$ \\
\hline \multirow{3}{*}{ Dimensión Planificación } & Muy Bajo & 5 & 5.2 & 3 & 3.1 \\
& Bajo & 10 & 10.4 & 12 & 12.5 \\
& Promedio & 67 & 69.8 & 69 & 71.9 \\
& Muy Alto & 14 & 14.6 & 12 & 12.5 \\
\hline \multirow{3}{*}{ Dimensión Autorregulación } & Muy Bajo & 3 & 3.1 & 3 & 3.1 \\
& Bajo & 16 & 16.7 & 14 & 14.6 \\
& Promedio & 69 & 71.9 & 67 & 69.8 \\
& Muy Alto & 8 & 8.3 & 12 & 12.5 \\
\hline \multirow{3}{*}{ Dimensión Evaluación } & Muy Bajo & 1 & 1.0 & 3 & 3.1 \\
& Bajo & 9 & 9.4 & 10 & 10.4 \\
& Promedio & 68 & 70.8 & 68 & 70.8 \\
& Muy Alto & 18 & 18.8 & 15 & 15.6 \\
\hline
\end{tabular}

\section{Comparación de promedios en la conciencia metacognitiva de acuerdo con el género y su relación con el rendimiento académico}

Adicionalmente al estudio de la evaluación de la conciencia metacognitiva y de sus dimensiones en la muestra de estudio, se buscó establecer si existían diferencias tanto por género y buscar relación con el rendimiento académico (medido a través de las notas promedio obtenidas del total de las asignaturas al final del semestre).

En cuanto al género no se encuentran diferencias significativas $(\mathrm{p}<.01)$ en los promedios (medido a través de la t de Student). Tampoco se encontró correlación significativa entre la conciencia metacognitiva con el rendimiento académico de los evaluados. Esto puede ser apreciado en la tabla 6.

\section{Tabla 6.}

Relación entre los puntajes de la conciencia metacognitiva y las notas promedio en la muestra de estudio $(n=96)$

\begin{tabular}{lllll}
\cline { 1 - 2 } & \multicolumn{2}{c}{ Pretest } & \multicolumn{2}{c}{ Pota Promedio } \\
& $\mathrm{r}$ & $\mathrm{sig}$ & $\mathrm{r}$ & $\mathrm{sig}$ \\
\hline Conciencia Metacognitiva & 0.12 & 0.25 & 0.01 & 0.91 \\
Planificación & 0.16 & 0.11 & 0.01 & 0.99 \\
Autorregulación & 0.10 & 0.35 & 0.04 & 0.68 \\
Evaluación & 0.08 & 0.41 & 0.02 & 0.88 \\
\hline *: p<.05: correlación significativa & & & &
\end{tabular}




\section{Discusión}

La motivación de este estudio fue conocer la conciencia metacognitiva y sus dimensiones en una muestra de ingresantes a la Facultad de ingeniería y arquitectura de una universidad privada de la ciudad de Lima; así como conocer las oportunidades metacognitivas de planificación, autorregulación y evaluación que proporciona la enseñanza en la facultad de la referencia.

El propósito principal del presente estudio fue obtener información comparativa acerca de los niveles de la conciencia metacognitiva y sus dimensiones en el pre test y post test, así como de las diferencias en los puntajes obtenidos al inicio y al final del semestre en el primer ciclo de estudios. Igualmente, determinar las relaciones existentes con el rendimiento académico evidenciado por las notas promedio, en el total de las asignaturas, obtenidas al término del semestre académico.

Hemos encontrado resultados que ubican a la mayoría de los participantes en niveles promedio en la conciencia metacognitiva y sus componentes de planificación, autorregulación y evaluación, con pequeñas variantes en los niveles bajos y superiores, lo que indica que predomina adecuada cualidad para reflexionar sobre la propia cognición y probablemente mayor uso de estrategias metacognitivas. Estos resultados son semejantes a los encontrados por Pacheco (2012) en estudiantes de ingeniería civil de una universidad pública de Lima.

Los resultados antes mencionados son favorables con relación a los estudiantes de psicología de universidades privadas y estatales de la ciudad de Arequipa, que muestran altos porcentajes de niveles bajos de metacognición, con relativa mejora en la distribución en los niveles de valoración en autorregulación y evaluación (Arias et al. 2014) y con los niveles medio y bajo encontrados por Cantillo et al. (2014) en estudiantes colombianos de psicología, resultados que señalan que los alumnos muestran dificultades para reflexionar sobre su propia cognición.

En nuestros resultados no se hallaron diferencias significativas en los promedios de conciencia metacognitiva y sus componentes considerando el género, aunque se observó el caso que los promedios son ligeramente mayores en el post test de los participantes varones. Se encuentra cierta semejanza con los encontrados por Pacheco (2012), en que los estudiantes varones de ingeniería civil que se ubican en el nivel superior de metacognición, destacan, más que las mujeres. Contrariamente, a lo que esperaba Broc (2012) las diferencias por género en estudiantes españoles de bachillerato mostraron en las mujeres una cierta ventaja en variables metacognitivas. Estos pocos y contradictorios hallazgos informan que hay que indagar más respecto de la influencia del género en la conciencia metacognitiva y sus componentes.

Las diferencias significativas encontradas entre el pre test y el post test en la conciencia metacognitiva y las dimensiones de planificación y autorregulación, nos comunican que ha habido relativo cambio favorable en la tendencia de uso de estrategias metacognitivas que guardan relación con establecer objetivos y metas, asignar tareas, recursos y predecir resultados; así como supervisar o monitorear la marcha de la tarea verificando y revisando alguna estrategia empleada. Estos hallazgos probablemente podrían sugerir que en el aprendizaje del primer ciclo, los profesores han proporcionado ciertas oportunidades para el uso adecuado de estrategias metacognitivas de planificación y de autorregulación; al respecto, Arteta y Huaire (2016) afirman que tanto en los profesores y alumnos están presentes ideas de carácter intuitivo respecto de los procesos, las condiciones, los resultados involucrados en la enseñanza y el aprendizaje que guardan relación con las estrategias metacognitivas.

Los resultados hallados en la dimensión de evaluación que mostrara diferencias sin significación estadística entre el pre test y el post test, evidencia en los estudiantes poco reconocimiento y uso de estrategias metacognitivas que les sirva para verificar su proceso de 
aprendizaje durante y al final del mismo; al parecer, no se evalúa los resultados de una estrategia empleada en términos de su eficacia o bien falta desarrollar capacidades específicas para emitir juicios, retroalimentaciones, valoraciones, reconstrucciones que generen dichas estrategias. Téngase en cuenta que el estudio se ha realizado en una muestra de ingresantes, que recién habían terminado la educación secundaria, que iniciaron una experiencia académica nueva para ellos y que estaban adaptándose a una nueva rutina con mayor carga de trabajo académico.

Finalmente, frente a la suposición compartida con otros investigadores de que las estrategias metacognitivas se hacen más presentes y efectivas a medida que los estudiantes aumentan su pericia a nivel académico, los resultados que se evidencian en la actualidad difieren unos de otros. Aún más, en algunos casos hay relaciones muy claras entre los resultados académicos y la conciencia cognitiva que alcanzan significación $(\mathrm{p}=.001)$ y en otra ausencia de relación. Por otro lado, al margen del nivel de rendimiento académico y del estadístico utilizado la correlación se cumple al margen del tipo de estudios cursado.

También se han reportado contradicciones en los primeros ciclos de estudios, en que el nivel de conciencia metacognitiva de los alumnos no guarda paralelismo con el rendimiento académico y, en los estudiantes de los últimos ciclos que a medida que el rendimiento académico disminuye, desciende igualmente el nivel de conciencia metacognitiva.

En nuestro estudio no se mostró relación significativa entre la conciencia metacognitiva y sus dimensiones con el rendimiento académico. Es probable que esté influenciando factores de índole motivacional (motivación de logro), afectivo (autoestima), hábitos de estudios, entre otros. Otro factor interveniente podría ser la medida del rendimiento académico que se ha tomado como nota promedio final en base a todas las asignaturas cursadas durante el semestre. Alternativas a ello, podrían ser, orientarse a medir el rendimiento académico por áreas y cursos por separado, estudiar cuáles estrategias metacognitivas influencia en el logro académico o aplicar diseños de medida pre y post.

Los resultados que hemos encontrado son diferentes a los mostrados por Thornberry (2008), quien halló correlaciones positivas entre dichas variables en alumnos ingresantes a una universidad privada de Lima, aunque cabe señalar que dicha relación no logró ser predictiva, y también con los resultados de Escanero et al. (2013), que hallaron correlación significativa ( $p<$ $0,05)$ entre la planificación (metacognición) y la nota obtenida. Por su parte Broc (2012), estudió la influencia relativa que ciertas variables metacognitivas y volitivas ejercían sobre el rendimiento académico, encontró como predictores del rendimiento académico final a la preparación de exámenes y la motivación. Las diferencias significativas encontradas, por este autor, en el rendimiento académico final en función de la modalidad elegida (Ciencias versus Letras) mostró resultados a favor de la primera.

Esta investigación nos permite contar con datos importantes para conocer la actividad metacognitiva de un grupo de estudiantes universitarios de primer ciclo, más no para generalizar más allá de lo aquí descrito, pues los estudios que se vienen realizando no son concluyentes y difieren ente ellos; de ahí que resulta relevante continuar con estudios en esta misma línea de investigación que hagan posible la utilización de instrumentos de medición más potentes cuyos resultados puedan ser contrastados con otras metodologías.

Asimismo, los resultados aquí presentados pueden llevar a considerar necesario fomentar el desarrollo de procesos metacognitivos en el contexto universitario, para permitir a los estudiantes incorporar estas habilidades a sus procesos de aprendizaje y conducirlos a un verdadero ejercicio de conciencia y aprendizaje autónomo. De igual forma resulta pertinente el desarrollo de programas de entrenamiento metacognitivo, dirigidos a mejorar y potenciar la 
actividad metacognitiva de los alumnos a la vez que también involucre al docente cuyo rol es fundamental.

\section{Referencias}

Abdellah, R. (2015). Metacognitive awareness and its relation to academic achievement and teaching performance of pre-service female teachers in Ajman University in UAE. Procedia - Social and Behavioral Sciences 174, 560-567. doi: https://doi.org/10.1016/j.sbspro.2015.01.707

Arias, W., Zegarra, J., Velarde, O. (2014). Estilos de aprendizaje y metacognición en estudiantes de psicología de Arequipa. Liberabit, 20(2), 267-279. Recuperado de http://ojs3.revistaliberabit.com/index.php/Liberabit/article/view/287

Arteta, H., \& Huaire, E. (2016). Estrategias metacognitivas y concepciones de aprendizaje en estudiantes universitarios. Horizonte de la Ciencia, 6(11), 149-158. doi: https://doi.org/10.26490/uncp.horizonteciencia.2016.11.236

Broc, M.A. (2012). Influencia relativa de variables metacognitivas y volitivas en el rendimiento académico de estudiantes de Bachillerato. Revista Española de Orientación y Psicopedagogía, 23(3), 63-80. doi: https://doi.org/10.5944/reop.vol.23.num.3.2012.11462

Bogdanović, I., Obadović, D., Cvjetićanin, S., Segedinac, M., \& Budić, S. (2015). Students' metacognition awareness and physics learning efficiency and correlation between them. European Journal of Physics Education, 6(2), 18-30. Recuperado de http://www.eujournal.org/index.php/EJPE/article/view/4/4

Bogantes, J., \& Palma K. (2016). La regulación continua de la enseñanza y del aprendizaje desde el evaluar para aprender. Una experiencia de la cátedra didáctica del lenguaje. Innovaciones Educativas, 18(24). 59-72. Recuperado de https://dialnet.unirioja.es/servlet/articulo?codigo $=5607285$

Burón, J. (1993). Enseñar a Aprender: Introducción a la metacognición (6º ed.). Bilbao: Mensajero.

Campo, K., Escorcia, D., Moreno, M., \& Palacio, J. (2016). Metacognición, escritura y rendimiento académico en universitarios de Colombia y Francia. Avances en Psicología Latinoamericana, 34(2), 233-252. doi: http://dx.doi.org/10.12804/apl34.2.2016.03

Cantillo, K., De la Hoz, A., \& Cerhiaro, E. (2014). Actividad metacognitiva en estudiantes universitarios: un estudio preliminar. Revista Psicología desde el Caribe. 31(3), 455-474, Recuperado de http://rcientificas.uninorte.edu.co/index.php/psicologia/article/view/5399

Córdoba, D., \& Marroquín, H. M. (2018). Mejoramiento del rendimiento académico con la aplicación de estrategias metacognitivas para el aprendizaje significativo. Revista UNIMAR, 36(1), 15-30. doi: https://doi.org/10.31948/unimar.36-1.1

Córdova, F., \& García, A. (2011). Adaptación del Inventario de Conciencia Metacognitiva. Lima: Universidad de San Martín de Porres.

Doganay, A., \& Demir, O. (2011). Comparison of the level of using metacognitive strategies during study between high achieving and low achieving prospective teachers. Educationat Sciences: Theory \& Practice, 11(4), 2036-2043. Recuperado de https://eric.ed.gov/?id=EJ962687

Escanero, J., Soria, S., Escanero, E., \& Guerra, M. (2013). Influencia de los estilos de aprendizaje y metacognición en el rendimiento académico de estudiantes de fisiología. Fundación Educación Médica, 16(1), 23-29. doi: http://dx.doi.org/10.4321/S201498322013000100005

Escorcia, D., \& Fenouillet, F. (2011). Quel rôle de la métacognition dans les performances en écriture?: Analyse de la situation d'étudiants en sciences humaines et sociales. Revue Canadienne de L'éducation, 34(2), 53-76. Recuperado de https://www.jstor.org/stable/canajeducrevucan.34.2.53 
Flavell, J. H. (1979). Metacognition and cognitive monitoring: a new area of cognitive developmental inquiry. American Psychologist, 34(10), 906-911. Recuperado de https://pdfs.semanticscholar.org/ee65/2f0f63ed5b0cfe0af4cb4ea76b2ecf790c8d.pdf

Guerrero G. (2017). Estrategias metacognitivas de lectura del nivel inferencial en la comprensión de textos argumentativos. (Tesis de maestría). Universidad Nacional Mayor de San Marcos, Lima, Perú. Recuperado de http://cybertesis.unmsm.edu.pe/handle/cybertesis/6468

Jiménez, V., Puente, A., Alvarado, J., Arrebillaga, L., \& Durante, L. (2009). Medición de estrategias metacognitivas mediante la Escala de Conciencia Lectora: ESCOLA. Electronic Journal of Research in Educational Psychology, 7(18), 779-804. doi: http://dx.doi.org/10.25115/ejrep.v7i18.1326

Huertas, A., Vesga, G., \& Galindo, M. (2014). Validación del instrumento Inventario de Habilidades Metacognitivas (MAI) con estudiantes colombianos. Praxis \& Saber. Revista de Investigación y Pedagogía, 5(10), 55-74. https://doi.org/10.19053/22160159.3022

Hernández, A., \& Camargo, A. (2017). Autorregulación del aprendizaje en la educación superior en Iberoamérica: Una revisión sistemática. Revista Latinoamericana de Psicología, 49, 146-160. doi: https://doi.org/10.1016/j.rlp.2017.01.001

Martínez, J. R. (2004). Concepción de aprendizaje, metacognición y cambio conceptual en estudiantes universitarios de psicología. (tesis de doctorado). Universidad de Barcelona. Barcelona, España. Recuperado de https://www.tdx.cat/bitstream/handle/10803/2632/Tesis_final.pdf?sequence=1

Mccann, E., \& Turner, J. (2004). Increasing student learning through volitional control. Teachers College Record, 106(9), 1695-1714. Recuperado de http://www.sfu.ca/ jcnesbit/EDUC220/ThinkPaper/MccannTurner2004.pdf

Meza, A., \& Lazarte, C. (2007). Manual de estrategias para el aprendizaje autónomo y eficaz. Lima: Universidad Ricardo Palma, Editorial Universitaria.

Moreano R., \& Roca, M. (2014). Estrategias cognitivas y metacognitivas de composición escrita que se promueven en un curso de redacción en una universidad privada de Lima (tesis de maestría), Pontificia Universidad Católica, Lima, Perú. Recuperado de http://hdl.handle.net/20.500.12404/5771

Naigeboren, M., \& Caram, G. (2004). Estrategias de aprendizaje y procesos metacognitivos en alumnos universitarios. XI Jornadas de Investigación. Recuperado de https://www.aacademica.org/000-029/109

O’Neil, H., \& J. Abedi (1996). Reliability and validity of a state metacognitive Inventory: Potential for alternative assessment. The Journal of Educational Research, 89(4), 234-45. Recuperado de https://www.jstor.org/stable/27542038

Osses, S., \& Jaramillo, S. (2008). Metacognición: un camino para aprender a aprender. Estudios Pedagógicos, 34(1), 187-197. doi: http://dx.doi.org/10.4067/S0718-07052008000100011

Owo, W., \& Ikwut, F. (2015). Relationship between metacognition, attitude and academic achievement of secondary school chemistry students in port Harcourt, Rivers State. Journal of Research \& Method in Education (IOSR-JRME), 5(6), 6-12. Recuperado de http://dx.doi.org/10.9790/7388-05630612

Pacheco, A. (2012). Estrategias metacognitivas y rendimiento en metodología del aprendizaje e investigación de los estudiantes de I ciclo de la Facultad Universidad Nacional de Ingeniería (Tesis de Maestría), Universidad Nacional Mayor de San Marcos, Lima, Perú. Recuperado de http://cybertesis.unmsm.edu.pe/handle/cybertesis/3433

Ramírez, J., Olarte, F., \& García, A. (2014). Rendimiento académico y aprendizaje autorregulado de estudiantes de ingeniería eléctrica y electrónica. Congreso Universitario de Innovación Educativa en las Enseñanzas Técnicas. Actas del XXI congreso universitario de innovación educativa en las enseñanzas técnicas. Valencia: Universidad Politécnica de Valencia, 2013, p. 200-213. Recuperado de http://hdl.handle.net/2117/21824 
Ramírez, J., Olarte, F., \& García, A. (2014). Estrategias de aprendizaje usadas por estudiantes de ingeniería eléctrica e ingeniería electrónica de primer semestre. Revista Educación en Ingeniería, 9(18), 216-227. doi: https://doi.org/10.26507/rei.v9n18.476

Shcraw, G., \& Denninson, R. (1994). Assessing metacognitive awareness. Contemporary Educational Psychology, 19(4), 460-475. doi: https://doi.org/10.1006/ceps.1994.1033

Sonowal, M., \& Alita, M. (2017). Metacognitive awareness and academic achievement of higher secondary level science stream students of dibrugarh distric, assam. International Education \& Research Journal, 3(6), 76-79. Recuperado de http://ierj.in/journal/index.php/ierj/article/view/1142

Thornberry, G. (2008). Estrategias metacognitivas, motivación académica y rendimiento académico en alumnos ingresantes a una universidad de Lima Metropolitana. Persona, 11, 177-193. Universidad de Lima. Recuperado de http://revistas.ulima.edu.pe/index.php/Persona/article/view/935

Weinstein, C. E., Palmer, D., \& Schulte, A. C. (1987). Learning and Study Strategies Inventory (LASSI). Clearwater, FL: $H \& H$ Publishing.

\section{Agradecimiento}

Se agradece el apoyo del psicólogo Augusto García Alvarado por facilitar el instrumento utilizado en la investigación. 